\title{
Méthode d'isolement et d'identification des résonances (M.I.I.R.) de cylindres et de tubes soumis à une onde acoustique plane dans l'eau
}

\author{
G. Maze et J. Ripoche \\ Université de Haute Normandie, Laboratoire d'Electronique et d'Automatique, groupe « Ultrasons », \\ U.E.R.S.T., B.P. 4006, 76077 Le Havre, France
}

(Recu le 7 décembre 1982, révisé le 24 février 1983, accepté le 7 mars 1983)

\begin{abstract}
Résumé. - Une nouvelle Méthode d' Isolement et d'Identification des Résonances (M.I.I.R.) de cylindres et de tubes élastiques immergés dans un fluide et soumis à des ultrasons rend possible, pour la première fois, l'isolement puis l'ídentification des résonances par des mesures du signal diffusé immédiatement après la fin de l'insonification par une onde acoustique plane. On enregistre notamment un spectre de rétrodiffusion de " quasi-raies ». Ce spectre de résonances est caractéristique du solide diffusant.
\end{abstract}

\begin{abstract}
A new Resonance Isolation and Identification Method (R.I.I.M.) is used, for the first time, to study elastic cylinders and shells imbedded in a fluid. It allows us the isolation and identification of resonances. The frequency spectrum of the backscattered echo, just after the excitation, is " quasi-linear ». This quasi-linear resonance spectrum characterizes the target and can be used for identification purposes.
\end{abstract}

\section{Introduction.}

Les premiers travaux que nous avons menés sur les ondes de surface [1-6] ont apporté une contribution à l'étude de la réflexion des ultrasons sur une interface métal-eau afin d'approcher un phénomène alors mal expliqué [7-12] : la diminution apparente du coefficient de réflexion, associée à un déplacement apparent du faisceau réfléchi et à un élargissement de ce faisceau pour un angle d'incidence un peu supérieur au deuxième angle critique. L'analyse électronique fine avec des transducteurs-récepteurs réalisés à cet effet a donné la possibilité de montrer que des ondes de surface, les ondes de type Rayleigh, se propageant à l'interface métal-eau, réémettent dans l'eau; cette réémission vient modifier la structure du faisceau « réfléchi » qui se propage dans l'eau quand le plan métallique est soumis à un faisceau ultrasonore limité. Une visualisation par une technique dite "Schlieren » et par une technique stroboscopique a également été mise en œuvre.

Afin d'approfondir la connaissance de ces ondes de surface, des techniques ont été imaginées [13-16] telles que celles utilisées maintenant par J. L. Izbicki $[17,18]$ afin de générer une onde de surface à l'interface métal-eau et afin de l'étudier, lors de sa propagation, à l'interface métal-air. Les méthodes optiques développées sont particulièrement adaptées :
- visualisation des surfaces d'équiphase des ondes ultrasonores se propageant à l'interface métal-air;

- étude du rayonnement d'un faisceau laser atteignant la surface et diffracté par les ondes de surface; elle conduit à la mesure des amplitudes des ondes, à l'analyse de la structure du faisceau, à l'examen des phénomènes non linéaires quand l'amplitude de l'onde de surface dépasse $80 \AA$ sur l'acier inoxydable, à la mise en évidence d'une focalisation.

Ces techniques ont été utilisées également pour étudier les ondes circonférentielles se propageant autour de cylindres [19-21].

Les ondes circonférentielles jouent un grand rôle dans les phénomènes de propagation au voisinage d'objets élastiques cylindriques immergés dans l'eau : les ondes de type Rayleigh, plus simplement appelées ondes de Rayleigh, et les ondes de "galerie à écho" (whispering gallery). Il existe d'autres ondes circonférentielles qui se propagent dans le fluide au voisinage de cibles : les ondes rampantes de Franz. Ces dernières ont également fait l'objet d'études [22]; leur contribution au signal diffusé, ou plus précisément rétrodiffusé, reste faible alors que les ondes de Rayleigh et de galerie à écho contribuent de façon caractéristique au signal rétrodiffusé par des corps élastiques.

Nous nous proposons, après un rappel des données théoriques, de présenter les résultats expérimentaux 
que nous avons obtenus dans l'étude de la rétrodiffusion par des cylindres et des tubes, puis de décrire la Méthode d'Isolement et d'Identification des Résonances (M.I.I.R.) que nous avons élaborée et mise au point.

\section{Diffusion des ondes acoustiques.}

La compréhension théorique des mécanismes qui gouvernent la diffusion des ondes acoustiques par des corps solides élastiques immergés dans l'eau a fait l'objet de nombreux travaux de $\mathrm{H}$. Überall et de ses collaborateurs [23-33]. A. Derem a présenté dans plusieurs articles [34-37], sous forme de synthèses, différents aspects de la théorie de la diffusion acoustique.

L'interprétation théorique des phénomènes observés [38-40] fait d'abord appel à une représentation de l'onde diffusée quand la longueur d'onde de l'onde incidente est de l'ordre de la circonférence du cylindre, c'est-à-dire quand on cherche la réponse acoustique aux basses fréquences. Le problème considéré est celui de la diffusion d'une onde acoustique plane de pulsation $\omega$ et de nombre d'onde $k_{1}=\omega / C_{1}\left(C_{1}\right.$ célérité acoustique dans l'eau) par une cible cylindrique métallique de rayon $a$ et de longueur infinie. Les célérités acoustiques des ondes à polarisation longitudinale et transversale sont respectivement $C_{\mathrm{L}}$ et $C_{\mathrm{T}}$. Le cylindre élastique de masse volumique $\rho_{2}$ est plongé dans l'eau de masse volumique $\rho_{1}$. La géométrie du problème est décrite sur la figure 1 .

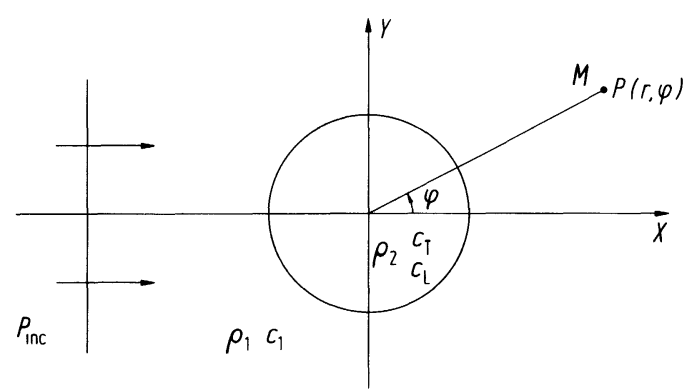

Fig. 1. - Géométrie du problème.

[Geometry of the cylinder scattering problem.]

L'onde de pression acoustique incidente plane peut se mettre sous la forme

$$
p_{\text {inc }}=P_{0} \exp j\left(k_{1} x-\omega t\right) .
$$

L'onde de pression diffusée s'obtient par application des conditions aux limites. La solution du problème au point $M$ peut se mettre sous la forme d'une série de Rayleigh de modes normaux de vibration $[23,41]$ :

$$
p_{\text {diff }}=P_{0} \exp (-j \omega t) \sum_{n=0}^{\infty} f_{n}\left(x_{1} ; r, \varphi\right),
$$

le mode de vibration d'indice $n$ s'écrivant :

$$
f_{n}\left(x_{1} ; r, \varphi\right)=\varepsilon_{n} j^{n} \frac{D_{n}^{[1]}(\omega, a)}{D_{n}(\omega, a)} H_{n}^{(1)}\left(k_{1} r\right) \cos n \varphi \text {. }
$$

La fréquence "réduite " $x_{1}=k_{1} a$ est égale au rapport de la circonférence du cylindre à la longueur d'onde dans l'eau $\lambda_{1}\left(k_{1}=2 \pi / \lambda_{1}\right)$. Les quantités $D_{n}$ et $D_{n}^{[1]}$ sont deux déterminants d'ordre 3 dont les éléments sont donnés dans la référence [34] et le facteur de Neumann $\varepsilon_{n}$ vaut 1 si $n=0$ et 2 si $n>0$. Les résultats numériques sont habituellement présentés à l'aide de la fonction $\left|F_{\infty}\left(x_{1}, \varphi\right)\right|$ appelée fonction de forme en champ lointain :

$$
\left|F_{\infty}\left(x_{1}, \varphi\right)\right|=\frac{2}{\sqrt{\pi x_{1}}}\left|\sum_{n=0}^{\infty} \varepsilon_{n} \frac{D_{n}^{[1]}}{D_{n}} \cos n \varphi\right| .
$$

Cette fonction est examinée pour un angle azimutal $\varphi$ fixé. Quand $\varphi=180^{\circ}$, on obtient la réponse rétrodiffusée.

S'il s'agit maintenant de manchon cylindrique dont la cavité interne est remplie d'un fluide (air, par exemple) le mode de vibration d'indice $n$ s'écrit :

$$
f_{n}\left(x_{1} ; r, \varphi\right)=\varepsilon_{n} j^{n} \frac{D_{n}^{[1]}(\omega, a, b)}{D_{n}(\omega, a, b)} H_{n}^{(1)}\left(k_{1} r\right) \cos n \varphi \text {. }
$$

$D_{n}^{[1]}$ et $D_{n}$ représentent alors deux déterminants d'ordre $6 ; a$ et $b$ sont respectivement les rayons externe et interne du manchon.

Lorsque la fréquence des ultrasons est élevée, il est plus avantageux de passer à une représentation asymptotique de l'onde diffusée, obtenue par application de la transformation de Sommerfeld-Watson à la série modale [25]. Les termes qui apparaissent alors sont directement associés aux ondes réfléchies, transmises et circonférentielles [26, 28]. Des progrès très sensibles ont été obtenus en utilisant le formalisme de « la matrice $S$ » (Scattering matrix) [31] très adaptée à la description de la diffusion des ondes acoustiques et conduisant à une caractérisation simple des résonances du diffuseur. On définit chaque résonance par le symbole $(n, l)$. En effet, il existe plusieurs résonances successives dans un mode normal de vibration d'indice $n$; il faut donc attribuer un indice supplémentaire $l=1,2, \ldots$ dans l'ordre où elles se présentent suivant les valeurs croissantes de $k_{1} a$ donc de la fréquence de l'onde acoustique incidente.

Une relation entre les ondes de surface élastiques et les résonances du cylindre a pu être établie $[30,34,35$, 42]. On a montré que les résonances des séries $l=1$, $l=2, l=3 \ldots$ sont produites respectivement par l'onde de Rayleigh notée $l=1$, par la première onde de galerie à écho $\left(\mathrm{GE}_{1}\right)$ notée $l=2$... Chaque onde se propageant à la périphérie du cylindre provoque l'apparition de la résonance $(n, l)$ dans le mode de vibration $n$. Les résonances sont la conséquence de la formation d'ondes stationnaires à la circonférence du cylindre à partir de deux ondes circonférentielles générées sous incidence critique et se propageant en sens contraire.

\section{Observation du spectre de rétrodiffusion.}

Pour observer un signal proportionnel au module de la fonction de forme au champ lointain, nous avons 
insonifié le cylindre uniformément avec un faisceau ultrasonore de diamètre supérieur à celui du cylindre (au moins quatre à six fois le diamètre du cylindre) [3840]. L'axe du faisceau est perpendiculaire à l'axe du cylindre.

Les calculs théoriques examinant le cas du régime permanent, il a fallu exciter le cylindre par un train de sinusoïdes (salve) comportant un nombre de sinusoïdes suffisant pour que le régime permanent soit atteint. Pour observer la rétrodiffusion, c'est-à-dire le signal diffusé pour $\varphi=180^{\circ}$, il faut disposer d'un transducteur-émetteur-récepteur (montage monostatique). En outre, pour explorer un domaine de $k_{1} a$ suffisant (de 0 à 100), il faut disposer de transducteurs à large bande passante.

L'étude expérimentale est conduite dans une cuve cylindrique de $3 \mathrm{~m}$ de diamètre et de $2 \mathrm{~m}$ de profondeur. La cuve est équipée de systèmes de positionnement précis pour les échantillons et les transducteurs. Un bras mobile dont la position angulaire est mesurée automatiquement permet de faire tourner un transducteur autour de l'échantillon. Les échantillons sont des cylindres d'aluminium de diamètre $(\varnothing=2 a)$ compris entre 4 et $15 \mathrm{~mm}$ et de longueur grande devant les longueurs d'onde acoustique (la longueur des cylindres est égale, au moins, à vingt fois leur diamètre). Ils sont plongés dans l'eau à la température ambiante. Leurs caractéristiques physiques sont celles retenues pour effectuer les calculs théoriques.

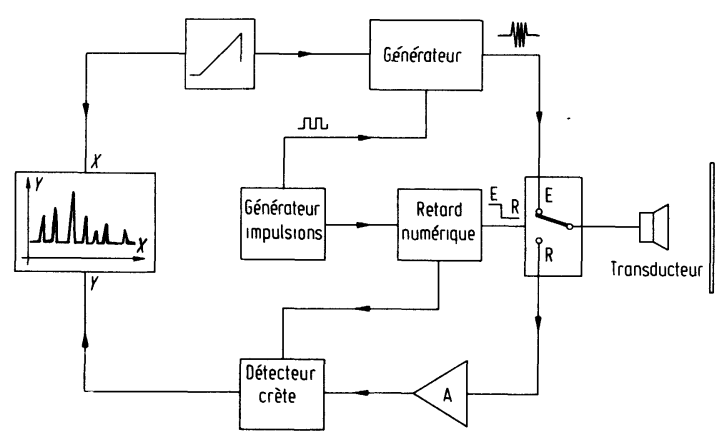

Fig. 2. - Système électronique.

[Block diagram of experimental apparatus.]

La figure 2 décrit le système électronique de mesure. Il est constitué d'un générateur de train de sinusoïdes dont il est possible de faire varier très lentement la fréquence en fonction du temps. Celle-ci est comprise entre 0,2 et $3 \mathrm{MHz}$ pour les mesures faites. L'expérience montre qu'avec un train d'ondes de 200 à $400 \mu \mathrm{s}$ le régime permanent est atteint. Il est toutefois prudent de ne pas allonger inconsidérément cette durée afin d'éviter les difficultés qui proviendraient des réflexions sur les parois de la cuve. Une porte analogique, commandée par un système à retard numérique permet de faire fonctionner le transducteur en émetteur ou en récepteur. Le signal reçu est amplifié puis traité par un détecteur-crête et enregistré sur une table traçante $X-Y$ en fonction de la fréquence.

Il s'agit d'enregistrer :

- la pression de l'onde acoustique diffusée par le cylindre en fonction de la fréquence dans une direction fixée. Quand il s'agit de rétrodiffusion $\varphi=180^{\circ}$ (montage monostatique);

- la pression de l'onde acoustique diffusée en fonction de l'angle azimutal $\varphi$, cette mesure s'effectuant pour une fréquence fixée (montage à deux transducteurs). Nous donnons sur la figure 3 le spectre de rétrodiffusion théorique ( $3 a)$ et celui enregistré $(3 b)$ après avoir fait les corrections pour tenir compte de la réponse en fréquence du transducteur.
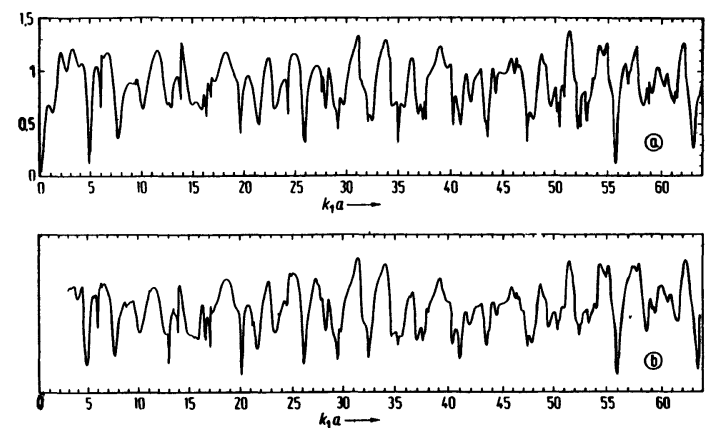

Fig. 3. - Spectre de rétrodiffusion d'un cylindre d'aluminium $: a$ ) théorique, $b$ ) expérimental.

[Frequency spectrum of backscattered echo from aluminium cylinder : $a$ ) theoretical spectrum, b) experimental spectrum.]

Les résultats obtenus font apparaître un accord remarquable entre la courbe de réponse mesurée expérimentalement et celle fournie par la théorie. La plupart des résonances ont pu être caractérisées. Sans entrer dans le détail de la discussion de ces spectres de rétrodiffusion, remarquons que de nombreuses résonances se manifestent le plus souvent par des minima dans les spectres de rétrodiffusion [40].

Il est possible en outre, d'enregistrer un signal proportionnel à la fonction de forme en champ lointain en utilisant un deuxième transducteur servant de récepteur et tournant autour du cylindre à une distance constante. Dans ce cas, la fréquence réduite $k_{1} a$ est fixée. Le premier transducteur continue à servir d'émetteur (montage à deux transducteurs).

Nous donnons sur, la figure 4 un exemple, parmi les résultats obtenus, pour $k_{1} a=4,89$.

De nombreux autres diagrammes angulaires, enregistrés pour des $k_{1} a$ plus grands $\left(k_{1} a=22 ; 5 ; 31,2\right.$; $55,7)$ ont été comparés à ceux obtenus par le calcul. L'accord est, en général, excellent même pour des diagrammes très complexes obtenus pour des $k_{1} a$ élevés. Cet accord persiste, que la fréquence soit une fréquence de résonance ou non. Ces travaux ont été discutés dans le même article [40]. 

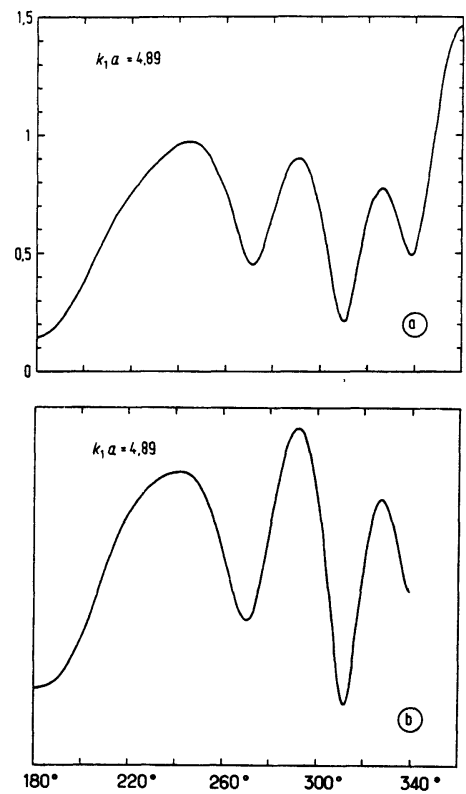

Fig. 4. - Diagramme angulaire pour $k_{1} a=4,89: a$ ) théorique, b) expérimental.

[Distribution in angle for $k_{1} a=4.89: a$ ) theoretical diagram, $b$ ) experimental diagram.]

\section{Méthode d'isolement des résonances. Spectres des résonances.}

Les enregistrements des spectres de rétrodiffusion pour un angle azimutal de $180^{\circ}$ et des diagrammes angulaires à une fréquence fixée qui ont fait l'objet du paragraphe précédent sont effectués lorsque le régime permanent est parfaitement instauré. Le détecteur-crête mesure l'amplitude du signal reçu à un certain instant au cours de la réception du signal diffusé. Cet instant est repéré par A sur la figure 5. Un train de sinusoïdes en provenance du transducteurémetteur, de durée $t_{\mathrm{ex}}$ (comprise entre 200 et $400 \mu \mathrm{s}$ ) génère dans le cylindre diverses propagations contribuant au signal diffusé. Celui-ci est reçu par le même transducteur servant alors de récepteur après avoir parcouru dans l'eau la distance cylindre-transducteur de l'ordre de $0,5 \mathrm{~m}$. La figure 5 représente des signaux reçus par le transducteur dans le cas où la fréquence de l'onde acoustique du faisceau incident (fréquence d'excitation) est éloignée d'une fréquence de résonance (Fig. $5 a$ ) et dans le cas où la fréquence d'excitation est très exactement égale à une fréquence de résonance (Fig. 5b). Dans le premier cas, le signal reçu a une enveloppe qui correspond bien à celle du train de sinusoïdes du faisceau incident de durée $t_{\mathrm{ex}}$. Dans le deuxième cas, on observe un régime transitoire suivi d'un régime permanent ; l'amplitude se fixe à une valeur inférieure à celle existant hors de toute résonance, une résonance se manifestant habituellement par un minimum; c'est dans cette région que se trouve positionné le détecteur-crête pour enregistrer les courbes des figures 3 et 4 ; on observe enfin après un

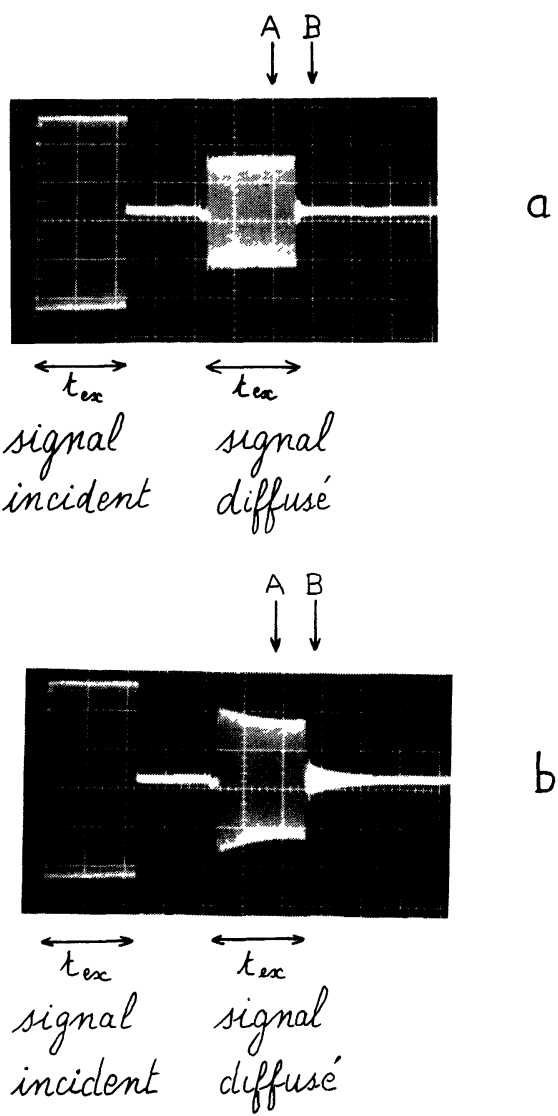

Fig. 5. - Signal rétrodiffusé (sur l'échelle horizontale, une division représente $100 \mu \mathrm{s}$ ) : a) hors résonance, $b$ ) à une fréquence de résonance.

[Backscattered echo (the horizontal scale is $100 \mu$ s per division) $: a$ ) far from a resonance, $b$ ) at a resonance.]

temps $t_{\mathrm{ex}}$ (durée de l'excitation forcée) un deuxième régime transitoire.

A l'aide d'un dispositif électronique convenable, il est possible de choisir la région " pointée » par le détecteur-crête pour qu'il fonctionne seulement lors $\mathrm{du}$ deuxième régime transitoire, environ $30 \mu$ s après la fin de l'excitation forcée. Cet instant est repéré par B sur la figure 5. Nous enregistrons alors, en fonction de la fréquence un signal rétrodiffusé après la fin de l'insonification. Le spectre obtenu est un spectre très finement résolu comportant des « quasi-raies » très marquées qui correspondent aux minima de la courbe de rétrodiffusion enregistrée par la méthode du paragraphe 3. Ainsi, les résonances du cylindre se trouvent parfaitement détectées tant que les résonances ne sont pas trop rapprochées $[43,44]$, ce qui est le cas dans un domaine de $k_{1} a$ compris entre 0 et 40 . La position du détecteur-crête, dans le second régime transitoire, a une incidence sur l'amplitude absolue et relative des " pics » des résonances, mais n'affecte pas la fréquence; une étude des amplitudes a été menée dans un article précédent [43].

La figure 6 donne un exemple des résultats obtenus pour un cylindre d'aluminium. Afin de comparer 


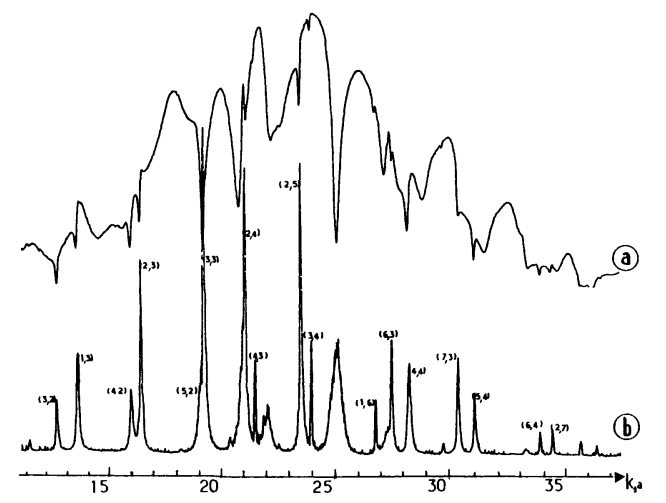

Fig. 6. - Cylindre d'aluminium : $a$ ) spectre de rétrodiffusion (régime permanent), $b$ ) spectre des résonances ( 2 éegime transitoire).

[Aluminium cylinder : a) backscattered spectrum (steady state response), $b$ ) resonance spectrum (second transient response). 1

aisément les deux spectres, nous donnons sur la même figure, l'enregistrement du signal rétrodiffusé dans le régime permanent et l'enregistrement du signal rétrodiffusé pendant le régime transitoire suivant la fin de l'insonification. Ces courbes doivent, ensuite, être corrigées pour tenir compte de la courbe de réponse en fréquence des transducteurs. Il est possible de faire une correction point par point ou mieux d'utiliser un système à microprocesseur pour effectuer une correction automatique [45]. Deux possibilités peuvent se présenter : aucune résonance ne s'est formée, le détecteur-crête n'enregistre pas de signal; une résonance s'est établie, un régime de vibrations dont l'amplitude décroît exponentiellement en fonction du temps apparaît, le détecteur-crête enregistre alors un signal. Lorsqu'il y a apparition d'une résonance, le cylindre continue à émettre un signal après l'insonification.

Pour un tube d'aluminium plein d'air, on observera un spectre moins riche en résonances dans le même domaine de $k_{1} a$. La figure 7 donne les résultats

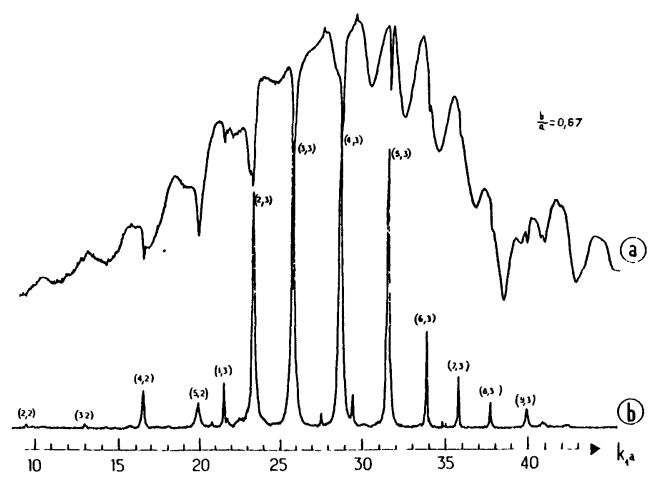

Fig. 7. - Tube d'aluminium plein d'air $(b / a=0,67): a)$ spectre de rétrodiffusion (régime permanent), $b$ ) spectre des résonances ( $2 \mathbf{e}$ régime transitoire).

[Aluminium cylindrical shell filled with air $(b / a=0.67)$ : a) backscattered spectrum (steady state response), b) resonance spectrum (second transient response).] obtenus pour un tube d'aluminium plein d'air dont le rapport $b / a$ est égal à 0,67 ( $b$ rayon interne, $a$ rayon externe). L'analyse détaillée de ses mesures et leur interprétation ont été faites récemment [46, 47].

Des cylindres et tubes de matériaux élastiques différents donnent des spectres du même type mais les résonances sont situées à des fréquences différentes. La figure 8 donne les résultats obtenus pour un tube de cuivre $(b / a=0,80)$.

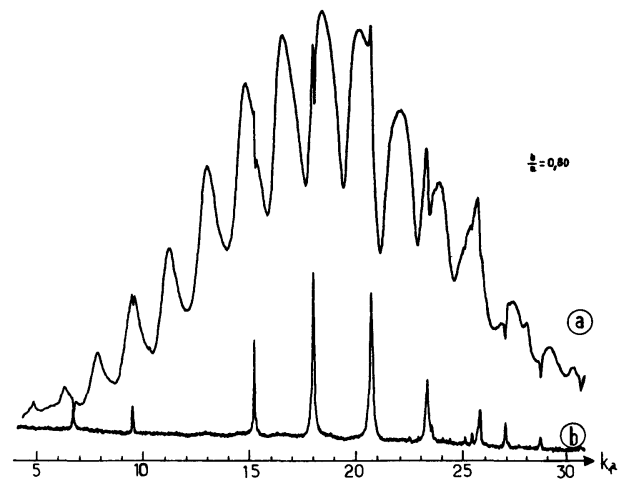

Fig. 8. - Tube de cuivre plein d'air $(b / a=0,80): a)$ spectre de rétrodiffusion (régime permanent), $b$ ) spectre des résonances ( $2^{\mathrm{e}}$ régime transitoire).

[Copper cylindrical shell filled with air $(b / a=0.80)$ : $a$ ) backscattered spectrum (steady state response), b) resonance spectrum (second transient response).]

Nous remarquons qu'il est devenu facile d'isoler les résonances sans être gêné par le fond non résonnant du spectre de rétrodiffusion obtenu par la technique décrite au paragraphe 3 . Le spectre de résonance ainsi enregistré peut remplacer la fonction de forme en champ lointain pour déterminer les résonances et obtenir une signature de la cible.

Nous venons de décrire une Méthode d'Isolement des Résonances qui constitue la première étape de la M.I.I.R.

\section{Identification des résonances.}

Les diagrammes angulaires que nous avons examinés au paragraphe 3 en fonction de l'angle azimutal $\varphi$, au cours du régime permanent et pour une fréquence fixée deviennent très complexes et comportent de nombreux lobes dès que la fréquence réduite $k_{1} a$ s'élève, bien que la coïncidence avec la théorie persiste [40].

En gardant la même technique avec deux transducteurs, nous mesurons l'amplitude du signal réemis par le cylindre après la fin de l'insonification forcée à une fréquence d'excitation précisément égale à une fréquence de résonance. Le détecteur-crête est positionné comme précédemment dans le deuxième régime transitoire, quelque $30 \mu \mathrm{s}$ après la fin de l'insonification forcée.

$\mathrm{La}$ rotation du transducteur-récepteur conduit à l'enregistrement d'un diagramme angulaire particu- 
lièrement simple, même si des résonances se trouvent très rapprochées. Ces diagrammes comportent un nombre pair de maxima et minima répartis régulièrement autour du cylindre. La figure 9 donne deux exemples relatifs à un cylindre d'aluminium, la figure $9 a$ pour un $k_{1} a$ correspondant à la résonance $(2,3)$ $(n=2 ; l=3)$ soit $k_{1} a=16,3$ et la figure $9 b$ pour un $k_{1} a$ correspondant à la résonance $(6,4)(n=6$, $l=4)$ soit $k_{1} a=33,9$.
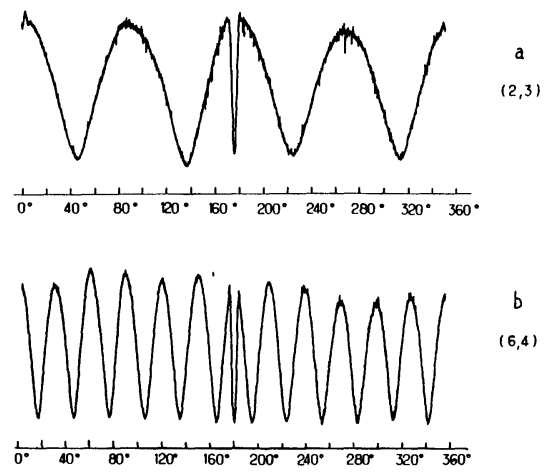

Fig. 9. - Cylindre d'aluminium. Diagrammes angulaires d'identification : a) $\left.k_{1} a=16,3 ;(n, l)=(2,3) ; b\right) k_{1} a=$ 33,$9 ;(n, l)=(6,4)$.

[Aluminium cylinder. Angle diagrams for identification : a) $\left.k_{1} a=16.3 ; \quad(n, l)=(2,3) ; \quad b\right) k_{1} a=33.9 ; \quad(n, l)=$ $(6,4)$.]

Quand le transducteur-récepteur effectue un tour complet, il apparaît sur la figure $9 a$ quatre maxima et quatre minima qui correspondent à des ventres et des nœuds de vibration sur le cylindre : quand le transducteur-récepteur passe devant un ventre de vibration situé sur le cylindre, il traverse un faisceau ultrasonore (c'est celụi qui subsiste après la fin de l'insonification) et le signal est maximal; quand le transducteurrécepteur passe devant un nœud de vibration, il ne traverse aucun faisceau ultrasonore, le signal est minimal. Les ventres et les nœuds de vibration sont dus à l'établissement, sur le cylindre, d'une onde stationnaire qui provient de la propagation de deux ondes circonférentielles générées sur le cylindre et se propageant en sens contraire : cette propagation de deux ondes circonférentielles se trouve ainsi confirmée expérimentalement. Les ondes circonférentielles, responsables de la résonance continuent à tourner sur le pourtour du cylindre jusqu'à ce qu'elles aient réémis toute leur énergie.

Le nombre de maxima permet de déterminer immédiatement le nombre $n$ fixant le mode de vibration. Ce nombre $n$ n'est autre que le nombre de longueurs d'onde $\lambda_{l}^{0}$ de l'onde circonférentielle sur la circonférence du cylindre. Le nombre d'onde $k_{l}^{0}$ de la $l$-ième onde circonférentielle s'obtient par la relation $k_{l}^{0} a=n$ avec $k_{l}^{0}=2 \pi / \lambda_{l}^{0}$.

Dans le cas de la figure $9 a$, quatre maxima conduisent à $n=2$ et dans le cas de la figure $9 b$, douze maxima conduisent à $n=6$. Ainsi, on peut déterminer $n$ pour la deuxième onde de galerie à écho $\left(\mathrm{GE}_{2}\right.$ pour $l=3$ ) et pour la troisième onde de galerie à écho $\left(\mathrm{GE}_{3}\right.$ pour $\left.l=4\right)$.

L'identification des résonances peut se pratiquer également pour des tubes. La figure 10 en donne un exemple.

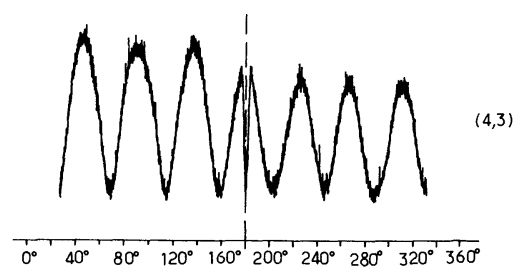

Fig. 10. - Tube d'aluminium plein d'air $(b / a=0,67)$. Diagramme angulaire d'identification. $k_{1} a=28,5 ;(n, l)=$ $(4,3)$.

[Aluminium cylindrical shell filled with air $(b / a=0.67)$. Angle diagram for identification. $k_{1} a=28.5 ;(n, l)=(4,3)$.]

D'autres exemples ont été donnés dans la communication [43] dans laquelle nous montrons que l'on peut séparer des résonances voisines pour $n=6,9,13$ correspondant à différentes ondes de galerie à écho dans un domaine de $k_{1} a$ compris entre 54 et 56. Remarquons sur la figure 9 , un minimum très marqué qui apparaît au milieu des diagrammes $\left(\varphi=180^{\circ}\right)$; il correspond au passage du transducteur-récepteur devant le transducteur-émetteur.

La deuxième étape de la M.I.I.R. donne donc le mode de vibration $n$.

Afin de s'assurer de la réalité des faisceaux ultrasonores et de leur génération par le cylindre après l'excitation, c'est-à-dire après la rupture du régime permanent, nous avons visualisé les faisceaux ultrasonores par la méthode "Schlieren" associée à la stroboscopie. Le nombre de faisceaux observés provenant du cylindre permet, là encore, de déterminer le nombre $n$ [48].

\section{Conclusion.}

Les travaux théoriques et les travaux expérimentaux avaient jusqu'alors conduit à l'obtention de spectres acoustiques habituellement donnés en fonction de la fréquence réduite $k_{1} a$. Ces spectres, bien que non finement résolus, comportent un fond non résonnant sur lequel se détachent des résonances plus ou moins marquées qui se manifestent, le plus souvent, par des minima dans le spectre de rétrodiffusion. La coïncidence entre les prévisions et l'expérience permettait déjà d'envisager une spectroscopie acoustique $[34,40$, 44, 45].

La Méthode d'Isolement et d'Identification des Résonances (M.I.I.R.) comporte deux étapes. Au cours de la première étape, nous nous intéressons au signal rétrodiffusé lors du régime transitoire suivant la fin de l'excitation. Dans la deuxième, toujours lors du même régime transitoire, nous nous intéressons 
au diagramme angulaire quand la fréquence d'excitation est égale à la fréquence d'une résonance particulière ; ce diagramme permet de fixer le mode de vibration $n$ concerné. Cette détermination est toujours possible en laboratoire, mais on ne peut l'utiliser dans des conditions de mesures difficiles qui ne permettent pas une rotation suffisante du transducteur-récepteur autour de la cible soumise aux ultrasons. Les mesures que nous avons effectuées s'avèrent relativement faciles à cause de la symétrie cylindrique des échantillons étudiés. Cependant, il est raisonnable de penser que cette deuxième étape, que nous appelons l'étape d'identification des résonances, puisse être utilisée dans d'autres conditions avec des échantillons tels que des sphères, des plaques, des structures diverses...

La première étape qui donne un spectre de rétrodiffusion de quasi-raies est, quant à elle, facile à mettre en œuvre si on dispose de l'électronique convenable. Elle donne un spectre de résonances finement résolu qui est une véritable signature de l'échantillon.

Les expériences que nous poursuivons sur des cylindres et des tubes constitués d'aluminium ou d'autres matériaux (cuivre par exemple) montrent que les travaux théoriques et expérimentaux permettent de dresser un catalogue de spectres. On pourra alors préciser la nature d'un échantillon de forme et de dimensions connues ou encore préciser la forme et les dimensions d'un échantillon de nature connue placé en milieu hostile. En effet, la position des résonances dépend de la forme (cylindre, tube, sphère, ellipsoïde...) de la cible diffusante et aussi des dimensions et de la nature du matériau.

Le spectre de rétrodiffusion des résonances obtenu par la M.I.I.R. peut être facilement comparé à un spectre étalon ou à un tableau de résonances préalablement relevées ou calculées. Dans un travail récent [49], il est proposé un modèle polynomial de signature ultrasonore. Ce modèle pourrait être utilisé dans une étape intermédiaire de calcul pour la reconnaissance des formes.

\section{Remerciements.}

Les auteurs remercient MM. B. Poirée (C.E.T.H.E.D.E.C.), A. Derem et J. L. Rousselot (L.C.T.) pour les échanges fructueux au cours des réunions de travail consacrées aux problèmes de la propagation acoustique.

Ce travail a été réalisé dans le cadre d'un contrat DRET (no 82/303).

Bibliographie

[1] Duclos, J., Ripoche, J., C.R. Hebd. Séan. Acad. Sci. 275B (1972) 525.

[2] Maze, G., Ripoche, J., C.R. Hebd. Séan. Acad. Sci. 278B (1974) 61.

[3] Maze, G., Duclos, J., Ripoche, J., Acustica 32 (1975) 181.

[4] Faure, A., Maze, G., Ripoche, J., C.R. Hebd. Séan. Acad. Sci. 280B (1975) 673.

[5] Faure, A., Maze, G., Ripoche, J., C.R. Hebd. Séan. Acad. Sci. 283B (1976) 45.

[6] Faure, A., MAZE, G., RIPOChe, J. Ultrasonics 14 (1976) 205.

[7] Sносн, A., Ergeb Exakten Naturwiss 23 (1950) 127.

[8] Mayer, W. G., J. Appl. Phys. 34 (1963) 909.

[9] Rollins, F. R. Jr., Appl. Phys. Lett. 7 (1965) 212.

[10] DiachoK, O. I., Technical Report $n^{\circ} 4$, Office of Naval Research (1969)

[11] Diachok, O. I., MaYer, W. G., J. Acoust. Soc. Amer. 47 (1970) 155.

[12] Neubauer, W. G., J. Appl. Phys. 48 (1973) 44.

[13] DiACHOK, O. I., Technical Report $n^{\circ} 5$, Office of Naval Research (1970).

[14] Faure, A., Maze, G., Ripoche, J., Phys. Lett. 58 (1976) 147.

[15] Maze, G., Faure, A., J. Opt. 9 (1978) 169.

[16] Faure, A., Maze, G., Rupoche, J., J. Physique Colloq. 40 (1979) C8-267.

[17] Izbicki, J. L., Maze, G., Faure, A., 4es Journées d'Etudes sur la Propagation Acoustique, Marseille, 1980, et Rev. CETHEDEC NS 80-1 (1980) 47.

[18] IzBicki, J. L., MAZe, G., Ripoche, J., 5es Journées d'Etudes sur la Propagation Acoustique, Le Havre, 1982, et Rev. CETHEDEC 72 (1982) 103.
[19] Faure, A., Maze, G., Ripoche, J., J. Appl. Phys. 48 (1977) 869.

[20] Faure, A., Maze, G., Ripoche, J., Phys. Lett. 53A (1975) 301.

[21] Faure, A., Maze, G., Ripoche, J., C.R. Hebd. Séan. Acad. Sci. 281B (1975) 385.

[22] Maze, G., Faure, A., Ripoche, J., Phys. Lett. 75A (1980) 214.

[23] Doolittle, R. D., Überall, H., J. Acoust. Soc. Amer. 39 (1966) 272

[24] Doolittle, R. D., Überall, H., Ugincius, P., J. Acoust. Soc. Amer. 43 (1968) 1.

[25] Ugincius, P., Überall, H., J. Acoust. Soc. Amer. 43 (1968) 1025.

[26] Frisk, G. V., Dickey, J. W., Überall, H., J. Acoust. Soc. Amer. 58 (1975) 996.

[27] Frisk, G. V., UUberall, H., J. Acoust. Soc. Amer. 59 (1976) 46.

[28] Dickey, J. W., Frisk, G. V., Überall, H., J. Acoust. Soc. Amer. 59 (1976) 1339.

[29] Überall, H., HuANG, H., Acoustical response of submerged elastic structures obtained though Integral Transforms dans : Physical Acoustics, Vol. XII (Academic Press, New York) 1976.

[30] Überall, H., Dragonette, L. R., Flax, L., J. Acoust. Soc. Amer. 61 (1977) 711.

[31] Flax, L., Dragonette, L. R.. Überall, H., J. Acoust. Soc. Amer. 63 (1978) 723.

[32] Diarmuid MuRPhy, J., BReITENBaCh, E. D., Überall, H., J. Acoust. Soc. Amer. 64 (1978) 677.

[33] Flax, L., Gaunaurd, G., Überall, H., Theory of resonance scattering, dans : Physical Acoustics, Vol. XV (Academic Press, New York) 1981. 
[34] Derem, A., Rev. CETHEDEC 58 (1979) 43.

[35] Derem, A., 4es Journées d'Etudes sur la Propagation Acoustique, Marseille, 1980, et Rev. CETHEDEC NS 80-1 (1980) 221.

[36] Derem, A., Rev. CETHEDEC 64 (1980) 1.

[37] Derem, A., 5es Journées d'Etudes sur la Propagation Acoustique, Le Havre, 1982 et Rev. CETHEDEC 72 (1982) 1.

[38] Faure, A., Maze, G., Ripoche, J., Rev. CETHEDEC 60 (1979) 175.

[39] Maze, G., Faure, A., Ripoche, J., 4es Journées d'Etudes sur la Propagation Acoustique, Marseille, 1980, et Rev. CETHEDEC NS 80-1 (1980) 251.

[40] Derem, A., Rousselot, J. L., Maze, G., Ripoche, J., FAURE, A., Acustica 50 (1982) 39.

[41] Faran, J. J., J. Acoust. Soc. Amer. 23 (1951) 405.

[42] Derem, A., Rousselot, J. L., Rev. CETHEDEC 67 (1981) 1 .
[43] Maze, G., Taconet, B., Ripoche, J., 8e Colloque sur le Traitement du Signal et ses Applications (GRETSI), Nice, 1981.

[44] Maze, G., Taconet, B., Ripoche, J., Phys. Lett. 84A (1981) 309.

[45] Taconet, B., Maze, G., 5es Journées d'Etudes sur la Propagation Acoustique, Le Havre, 1982, et Rev. CETHEDEC 72 (1982) 121.

[46] Rousselot, J. L., 5es Journées d'Etudes sur la Propagation Acoustique, Le Hayre, 1982, et Rev. CETHEDEC 72 (1982) 47.

[47] Maze, G., Taconet, B., Ripoche, J., 5es Journées d'Etudes sur la Propagation Acoustique, Le Havre, 1982, et Rev. CETHEDEC 72 (1982) 103.

[48] Maze, G., Ripoche, J., J. Acoust. Soc. Amer. 73 (1983) 41.

[49] Faure, A., Foubert, M., Ripoche, J., Maze, G., 2nd Symposium IASTED Applied Modelling and Simulation, juin 1982, Paris. 